\title{
Structure and vibrational dynamics of interfacial Sn layers in $\mathrm{Sn} / \mathrm{Si}$ multilayers
}

\author{
B. Roldan Cuenya* and W. Keune \\ Laboratorium für Angewandte Physik, Gerhard-Mercator-Universität, D-47048 Duisburg, Germany
}

W. Sturhahn, T. S. Toellner, and M. Y. Hu

Advanced Photon Source, Argonne National Laboratory, Argonne, Illinois 60439

(Received 12 April 2001; published 27 November 2001)

\begin{abstract}
The structure and vibrational dynamics of room-temperature-grown nanoscale $\mathrm{Sn} /$ amorphous $(a$-) Si multilayers have been studied by x-ray diffraction, Raman scattering, ${ }^{119} \mathrm{Sn}$ Mössbauer spectroscopy, and ${ }^{119} \mathrm{Sn}$ nuclear-resonant inelastic x-ray scattering (NRIXS) of synchrotron radiation. With increasing Sn-layer thickness, the formation of $\beta$-Sn was observed, except at the $\mathrm{Sn} / \mathrm{Si}$ interfaces, where a 10 - $\AA$-thick metastable pure amorphous- $\alpha$-Sn-like layer remains stabilized. By means of NRIXS we have measured the Sn-projected vibrational density of states (VDOS) in these multilayers (in particular, at the interfaces), and in 500- $\AA$-thick epitaxial $\alpha$-Sn films on $\operatorname{InSb}(001)$ as a reference. Further, the Sn-specific Lamb-Mössbauer factor ( $f$ factor), mean kinetic energy per atom, mean atomic force constant, and vibrational entropy per atom were obtained. The VDOS of the amorphous- $\alpha$-Sn-like interface layer is observed to be distinctly different from that of (bulk) $\alpha$-Sn and $\beta$-Sn, and its prominent vibrational energies are found to scale with those of amorphous Ge and Si. The observed small difference in vibrational entropy $\left(\Delta S / k_{B}=+0.17 \pm 0.05\right.$ per atom) between $\alpha$-Sn and interfacial amorphous- $\alpha$-like Sn does not account for the stability of the latter phase.
\end{abstract}

DOI: $10.1103 /$ PhysRevB.64.235321

PACS number(s): 68.60.-p, 61.43.Dq, 76.80.+y, 63.50. $+\mathrm{x}$

\section{INTRODUCTION}

Elemental semiconductors with diamond structure, e.g., $\mathrm{Si}$ or Ge, are known to exist in amorphous form when prepared either as bulk glasses or as thin films. ${ }^{1,2}$ An exception is gray tin $(\alpha-\mathrm{Sn})$ that in the crystalline state is a nonpolar semiconductor with diamond structure and a band gap nearly equal to zero $(0.08 \mathrm{eV}$ at $300 \mathrm{~K}){ }^{3}$ To the best of our knowledge only one literature report exists on the observation of the amorphous phase of $\alpha$-Sn, in this case produced by ion implantation. ${ }^{4}$ The amorphous structure of group IV semiconductors $(\mathrm{Ge}, \mathrm{Si})$ has been shown ${ }^{5}$ to deviate from the ideal tetrahedral coordination of the diamond structure by bending of the four nearest-neighbor covalent bonds, with a spread in bond angles of about $10^{\circ}$ around the ideal value of $109^{\circ}$. Deviations by bond lengthening are not observed to be more than $1 \% .^{5}$ At ambient pressure $\alpha$-Sn is the stable lowtemperature phase of bulk tin, which transforms into the metallic body-centered tetragonal $\beta$-Sn phase (with lattice parameters $a=5.83 \AA, c=3.18 \AA$ ) when the temperature is raised above $T_{c}=13.2^{\circ} \mathrm{C}$. The $\alpha \leftrightarrow \beta$ phase transition of bulk $\mathrm{Sn}$ is an entropy-driven structural transformation that is determined by the difference in vibrational entropy of the two phases. ${ }^{6}$ It has been shown that crystalline $\alpha$-Sn ( $a$ $=6.489 \AA$ ) can be stabilized as a metastable phase above $T_{c}$ [and, in particular, at room temperature (RT)] in the form of a thin film of considerable thickness $(\sim 1000 \AA)$ by heteroepitaxy on an appropriate substrate. For instance, epitaxial RT growth of $\alpha$-Sn films is feasible on $\operatorname{InSb}(001)$ substrates $(a=6.4798 \AA)$ because of close lattice matching. ${ }^{3,7-12}$ On the other hand, RT growth of epitaxial $\alpha$-Sn-type overlayers on $\mathrm{Si}(111)$ substrates has been observed to be limited to only $\sim 3$ atomic layers, because the larger lattice misfit ( $\mathrm{Si}: a=5.4309 \AA$ ) favors the transition to $\beta$-Sn above such low Sn coverages. ${ }^{13-15}$
In the present work we investigated $\operatorname{Sn}\left(t_{\mathrm{Sn}}\right) / \mathrm{Si}\left(t_{\mathrm{Si}}\right)$ multilayers of different $\mathrm{Sn}$ and Si thicknesses $\left(t_{\mathrm{Sn}}\right.$ and $t_{\mathrm{Si}}$, respectively). Contrary to the crystallographically well-ordered epitaxial systems mentioned above, the Sn layers in the present multilayers are embedded between amorphous-Si $(a-\mathrm{Si})$ layers. This poses the interesting question for the type of crystallographic structure of the Sn layers under this condition, in particular, in the $\mathrm{Sn} / \mathrm{Si}$ interfacial region. In general, knowledge of the interfacial structure is important for the detailed understanding of Schottky barriers in metal/ semiconductor systems. ${ }^{16} \mathrm{Sn} / \mathrm{Si}$ multilayers form an interesting system, where atomic interdiffusion at the $\mathrm{Sn} / \mathrm{Si}$ interfaces may be excluded, because Sn and Si do not form any solid solutions or compounds, even after melting, according to the thermodynamic phase diagram. ${ }^{17}$ The principal aim of our study was to gain insight into the structure and lattice dynamics of the Sn layers, in particular, in the $\mathrm{Sn} / \mathrm{Si}$ interfacial region.

We have employed the x-ray diffraction (XRD), Raman scattering, ${ }^{119} \mathrm{Sn}$ conversion-electron Mössbauer spectroscopy (CEMS), and ${ }^{119} \mathrm{Sn}$ nuclear-resonant inelastic x-ray scattering (NRIXS) of synchrotron radiation. NRIXS is an efficient and unique method for a direct measurement of the vibrational (or phonon) density of states (VDOS) of thin films and buried interfaces that contain Mössbauer isotopes independent of whether the structure is crystalline or amorphous. ${ }^{18-22}$ CEMS and NRIXS are local methods providing information on an atomic scale.

\section{EXPERIMENT}

${ }^{119} \mathrm{Sn} / \mathrm{Si}$ multilayers of composition $\left[\operatorname{Sn}\left(t_{\mathrm{Sn}}\right) / \mathrm{Si}\left(t_{\mathrm{Si}}\right)\right]_{n}$ were grown on $\mathrm{Si}(111)$ wafers in an ultrahigh vacuum (UHV) system, with average $\mathrm{Sn}$ thicknesses $\left(t_{\mathrm{Sn}}\right)$ between $0.4 \AA$ and $100 \AA$, and Si thicknesses $\left(t_{\mathrm{Si}}\right)$ between $20 \AA$ and 
$80 \AA$. (For the definition of the Sn layer thickness, see below). The number of bilayers, $n$, varies between 1 and 50 . The enrichment of ${ }^{119} \mathrm{Sn}$ was $82.9 \%$. The Si substrates were rinsed in acetone and ethanol just before being loaded into the UHV chamber. The $\mathrm{Si}(111)$ substrates were cleaned in situ by annealing for $15 \mathrm{~min}$ at $300^{\circ} \mathrm{C}$.

The multilayers were grown at two different deposition temperatures, $T_{s}$, i.e., $-50{ }^{\circ} \mathrm{C}$ and $\mathrm{RT}$, to investigate the effect of temperature on the $\alpha \rightarrow \beta$ phase transition. Highpurity (undoped) Si was evaporated by an electron gun with a deposition rate of $0.1-0.2 \AA / s$, and a deposition pressure of $(3-5) \times 10^{-9}$ mbar. At first a Si layer was deposited on the $\mathrm{Si}(111)$ substrates, and all multilayers were coated with $\mathrm{Si}$ on the top to avoid oxidation. It is known that UHV deposition of Si films under such conditions results in amorphous- $(a-) \mathrm{Si}$ layers. Metallic ${ }^{119} \mathrm{Sn}$ was evaporated from a Knudsen cell $\left(\mathrm{Al}_{2} \mathrm{O}_{3}\right.$ crucible) at a deposition pressure of $(1-2) \times 10^{-9}$ mbar and a deposition rate between $0.02-0.025 \AA / s$. The real substrate temperature during deposition was somewhat higher than the previously indicated values, since we measured up to $40^{\circ} \mathrm{C}$ by a thermocouple at the sample holder for the RT case.

The evaporation rates and film thicknesses were monitored with a calibrated quartz-crystal microbalance and were determined from the bulk density of $\beta$-Sn. All Sn layer thicknesses in the multilayers are given in equivalent $\beta$-Sn thicknesses ( $\beta$-Sn Ångstrom). For instance, an average ( $\beta$-Sn) thickness of $0.4 \AA$ (see above) corresponds to a coverage of $1.48 \times 10^{14} \mathrm{Sn}$ atoms $/ \mathrm{cm}^{2}$. We will demonstrate later that amorphous $\alpha$-like $\mathrm{Sn}$ is formed for $\mathrm{Sn}$ coverages $\lesssim 10 \AA$ ( $\beta$-Sn Ångstrom). If we had crystalline $\alpha$-Sn, this would correspond to a physical thickness of $10 \AA \times 1.27$ $=12.7 \AA$ of crystalline $\alpha$-Sn (the factor 1.27 is equal to the ratio of the mass densities of $\beta$-Sn and $\alpha$-Sn). However, since the mass density of amorphous- $\alpha$-like-Sn is unknown, we will use equivalent $\beta$-Sn thicknesses also for coverages $\lesssim 10 \AA$.

Additionally, x-ray $(\theta-2 \theta)$ reflectometry studies under grazing incidence geometry were performed to check the quality and thickness periodicity of the multilayers.

For comparison, we have also grown epitaxial 200-500 $\AA{ }^{119} \mathrm{Sn}(001)$ films (crystalline $\alpha$-Sn) on $\operatorname{InSb}(001)$ substrates, as described in Ref. 12. (Here the thickness is given in $\alpha$-Sn Ångstrom .)

${ }^{119} \mathrm{Sn}$ CEMS provided information on the structural phase of tin in the multilayers from the $\simeq 0.5 \mathrm{~mm} / \mathrm{s}$ difference in the isomer shifts of bulk $\alpha-\operatorname{Sn}(\delta=2.03 \pm 0.02 \mathrm{~mm} / \mathrm{s}$ at RT) and bulk $\beta$-Sn $(\delta=2.56 \pm 0.01 \mathrm{~mm} / \mathrm{s}$ at RT). Both isomer shift values are relative to a $\mathrm{BaSnO}_{3}$ or $\mathrm{CaSnO}_{3}$ standard absorber as described in Refs. 23 and 24. For the $23.88-\mathrm{keV}$ Mössbauer $\gamma$ radiation, ${ }^{119} \mathrm{Sn} *$ in a $\mathrm{CaSnO}_{3}$ matrix was used as source. Conversion electrons were detected in a $\mathrm{He}-4 \%$ $\mathrm{CH}_{4}$ gas proportional counter. The Mössbauer spectra were least squares fitted with the program NORMOS, ${ }^{25}$ either with a Lorentzian line shape or occasionally with a Voigt profile (convolution of a Lorentzian single line with a Gaussian), depending on the type of sample.

The NRIXS experiments were performed at the Advanced
Photon Source (Sector 3-ID undulator beamline), Argonne National Laboratory, USA. We observed the reemission of $\mathrm{x}$ rays from the $23.88-\mathrm{keV}$ nuclear resonance of ${ }^{119} \mathrm{Sn}$. The deexcitation of the nucleus by emission of a photon takes place on a time scale of the lifetime of the nuclear resonance. If the nucleus is excited by a pulsed synchrotron radiation, the discrimination of the nuclear-resonant absorption from the electronic contribution is efficiently done by counting only delayed photons. Tuning the energy of the incident synchrotron radiation with respect to the nuclear resonance, while monitoring the total yield of the fluorescence photons, gives a direct measure of the VDOS. ${ }^{19}$ The NRIXS measurements were done at RT in an experimental setup very similar to Fig. 6 in Ref. 26. A high resolution monochromator for the $23.88-\mathrm{keV}$ x-rays (nuclear-resonance energy of ${ }^{119} \mathrm{Sn}$ ) was used during these experiments. ${ }^{27}$ The energy resolution achieved was $\simeq 0.8 \mathrm{meV}$. From the VDOS of the Sn films in the multilayers, we will determine the recoilless factor $f$, mean kinetic energy per atom $\bar{T}$, mean force constant $\bar{K}$, and the vibrational entropy of the Sn layers.

Raman spectra were obtained with a Jobin-Yvon triple spectrometer (T64000) equipped with a liquid $N_{2}$-cooled charge-coupled-device detector, using $50 \mathrm{~mW}$ of $476 \mathrm{~nm}$ radiation from a $\mathrm{Kr}^{+}$laser. All spectra were obtained in a quasibackscattering geometry with the sample in a He atmosphere.

\section{RESULTS}

\section{A. X-ray reflectometry and diffractometry}

We have used small-angle $\mathrm{x}$-ray reflectometry to check the multilayer quality. Figure 1 shows typical reflectometry curves obtained from multilayers with thicknesses $\left(t_{\mathrm{Sn}} / t_{\mathrm{Si}}\right)$ of $1.6 \AA / 77 \AA, 15 \AA / 72 \AA$, and $30 \AA / 42 \AA$. In Fig. 1(a) and 1(b) we recognize a double periodicity. The oscillations with a small period (Kiessig fringes) correspond to the total thickness interference of the multilayer, ${ }^{28}$ that is to $\left(t_{\mathrm{Sn}}+t_{\mathrm{Si}}\right) x n$. The larger periodicity (superstructure Bragg peaks) observed in Figs. 1(a) and 1(b) reflects the bilayer thickness $\left(t_{\mathrm{Sn}}\right.$ $+t_{\mathrm{Si}}$ ) in the multilayer. ${ }^{29}$ One can see only one period (Kiessig fringes) in Fig. 1(c), because this sample consists of only one bilayer $(n=1)$.

The observation of Kiessig fringes demonstrates that the surfaces of the multilayers are nearly atomically flat. Since at least two superstructure Bragg peaks are detected, our samples are of good multilayer quality.

Figure 2 displays wide-angle $\mathrm{x}$-ray diffractometry results on $\mathrm{Sn} / \mathrm{Si}$ multilayers grown on $\mathrm{Si}(111)$ for $[\operatorname{Sn}(10 \AA) / \operatorname{Si}(50 \AA)]_{50}(\mathrm{a})$ and $[\operatorname{Sn}(20 \AA) / \operatorname{Si}(50 \AA)]_{25}$ (b), together with two scans showing $\beta$-Sn in $\operatorname{Sn}(1000 \AA) /$ $\operatorname{Si}(111)$ (c), and $\alpha$-Sn in $\operatorname{Sn}(200 \AA) / \operatorname{InSb}(001)$ (d), the latter being used as references. In Fig. 2(a) only the Bragg peaks of the $\mathrm{Si}(111)$ substrate clearly appear, but one can also notice a weak upward curvature of the background around $2 \theta$ $=40^{\circ}-50^{\circ}$ which includes the position of the (220) $\alpha$-Sn peak [Fig. 2(d)]. This is more clearly demonstrated in the inset of Fig. 2 (top), where the region near $40^{\circ}-50^{\circ}$ is magnified. The curved background near $40^{\circ}-50^{\circ}$ might be evi- 


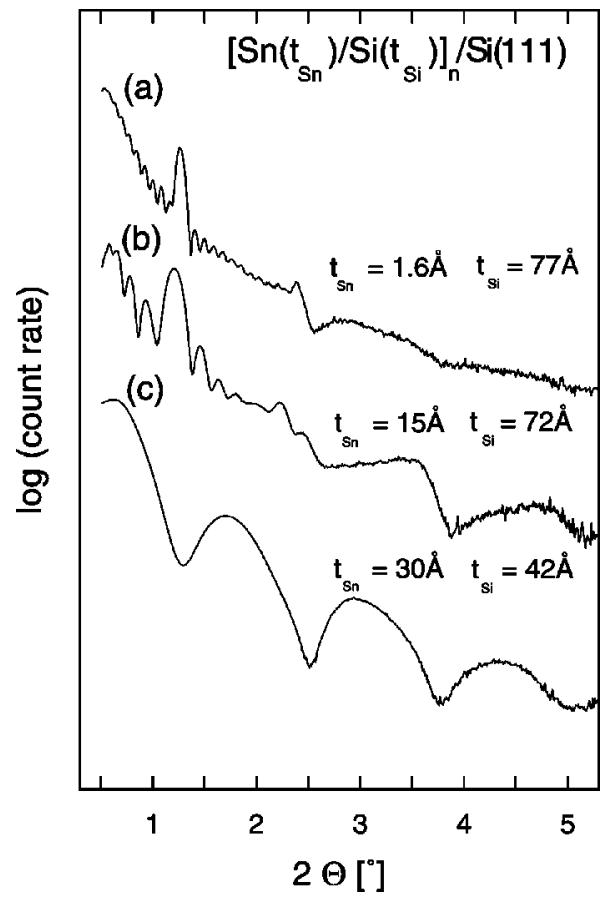

FIG. 1. Small-angle $x$-ray reflectometry scans $(\theta-2 \theta)$ measured in grazing incidence for (a) $\left[{ }^{119} \operatorname{Sn}(1.6 \AA) / \mathrm{Si}(77 \AA)\right]_{12}$, (b) $\left[{ }^{119} \operatorname{Sn}(15 \AA) / \operatorname{Si}(72 \AA)\right]_{6}$, and (c) $\left[{ }^{119} \operatorname{Sn}(30 \AA) / \operatorname{Si}(42 \AA)\right]_{1} .\left(\lambda_{K_{\alpha}}\right.$ $=1.54178 \AA$ A.)

dence for the presence of amorphous $\alpha$-like $\mathrm{Sn}$. In addition to the $\mathrm{Si}(111)$ substrate diffraction peaks, a small peak corresponding to the (400) $\beta$-Sn peak [Fig. 2(c)] was observed in Fig. 2(b), that is, $20 \AA \mathrm{Sn}$ in the $\mathrm{Sn} / \mathrm{Si}$ multilayers contain a contribution of $\beta$-Sn. As in the case of $[\operatorname{Sn}(10 \AA)$ / $\operatorname{Si}(50 \AA)]_{50}$, no $\alpha$-Sn peaks were observed in Fig. 2(b).

\section{B. Mössbauer spectroscopy}

We have measured the Sn thickness dependence of CEMS spectra at $\mathrm{RT}$ in $\left[\operatorname{Sn}\left(t_{\mathrm{Sn}}\right) / \operatorname{Si}(50 \AA)\right]_{n}$ multilayers to obtain information about the critical thickness at which the $\alpha$-type Sn to $\beta$-Sn phase transition occurs. All of these samples were deposited at RT. Figure 3 shows typical ${ }^{119} \mathrm{Sn}$ Mössbauer spectra. From the Lorentzian or Gaussian (Voigt profile) least-squares fit, we obtained the isomer shift, which is a useful parameter to distinguish the two possible tin phases ( $\alpha$ or $\beta$ ), as mentioned before. All isomer shift $\delta$ values are given relative to a $\mathrm{CaSnO}_{3}$ absorber at RT. (At RT, $\mathrm{CaSnO}_{3}$, $\mathrm{BaSnO}_{3}$, and $\mathrm{SnO}_{2}$ absorbers have the same isomer shifts ${ }^{24}$ ).

The Mössbauer spectra of Figs. 3(a)-3(d) could be fitted with a single line, resulting in an isomer shift very close to the bulk value of $\alpha$-Sn. ${ }^{23,24}$ Therefore, pure $\alpha$-like Sn exists up to $t_{\mathrm{Sn}}$ of $10 \AA$. Above $10 \AA$ of $\mathrm{Sn}$, two single lines were needed to fit the spectra, corresponding to the isomer shift of the $\alpha$-like Sn and $\beta$-Sn phases, respectively. The presence of $\beta$-Sn above $10 \AA$ contributes to a characteristic asymmetry in the peak shape [Figs. 3(e)-3(h)]. With increasing Sn layer thickness, the relative intensity of the $\beta$-Sn peak becomes larger, and at a thickness of $100 \AA$ [Fig. 3(i)], the $\beta$-Sn contribution dominates. These results demonstrate that

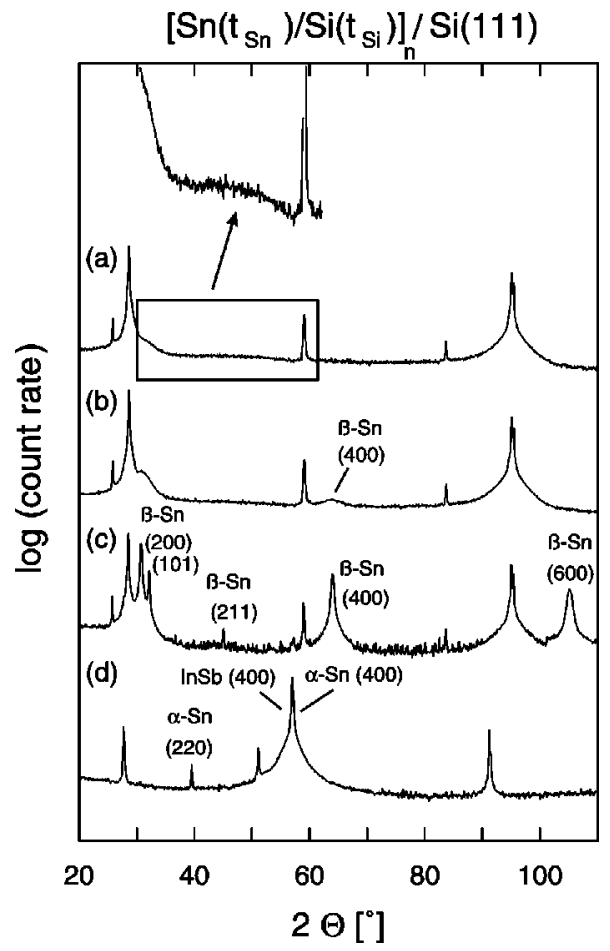

FIG. 2. Wide-angle x-ray diffractometry scans $(\theta-2 \theta)$ of: (a) $[\operatorname{Sn}(10 \AA) / \operatorname{Si}(50 \AA)]_{50} / \operatorname{Si}(111), \quad$ (b) $[\operatorname{Sn}(20 \AA) / \operatorname{Si}(50 \AA)]_{25} /$ $\mathrm{Si}(111)$; (c) epitaxially grown crystalline $\beta$-Sn with (200) and (101) texture in $\operatorname{Si}(70 \AA) / \operatorname{Sn}(1000 \AA) / \mathrm{Si}(111)$, and (d) epitaxial $\alpha$-Sn with predominant (400) orientation and some (220) orientation in $\mathrm{Si}(100 \AA) / \mathrm{Sn}(200 \AA) / \mathrm{InSb}(001)$. The observed Bragg peaks of $\alpha$-Sn in (d) and of $\beta$-Sn in (b), (c) are indicated. All other peaks observed in the figure belong to the corresponding substrates. $\left(\lambda_{K_{\alpha}}=1.54178 \AA\right)$.

$\alpha$-like $\mathrm{Sn}$ in these multilayers is only stable for very small thicknesses, less than or equal to $10 \AA$.

Table I(a) displays the Mössbauer parameters (isomer shift $\delta$ and linewidth $\Gamma$ ) of the $\operatorname{Sn}\left(t_{\mathrm{Sn}}\right) / \operatorname{Si}(50 \AA)$ multilayers deposited and measured at RT. For one of the samples, with $t_{\mathrm{Sn}}=10$, a least squares fitting with a Voigt profile for the $\alpha$-like $\mathrm{Sn}$ line resulted in a slightly better fitting of the corresponding spectrum.

Table I(b) shows the Mössbauer parameters (isomer shift and linewidth) of $\alpha$-Sn in $500 \AA$ epitaxial ${ }^{119} \mathrm{Sn}(001)$ on $\mathrm{InSb}(001)$ and of a $\beta$-Sn foil, both measured at RT, in comparison with the $\alpha$ - and $\beta$-Sn bulk values taken from the literature. ${ }^{24,23}$ One can notice that the isomer shift values of the $\alpha$-like Sn phase in the multilayers of Table I(a) are very close to those of bulk $\alpha$-Sn and epitaxial $\alpha$-Sn [Table I(b)].

Analysis of the Mössbauer spectra (Fig. 3) yields the relative contributions of the $\alpha$-like $\mathrm{Sn}$ and $\beta$-Sn phases to the total spectral area. The measured relative spectral area $A_{\alpha}$ of the $\alpha$-like phase as a function of the individual Sn thickness $t_{\mathrm{Sn}}$ in our multilayers is plotted in Fig. 4 (full squares). Up to $t_{\mathrm{Sn}}=10 \AA$ only $\alpha$-like Sn exists, while with increasing $t_{\mathrm{Sn}}$ the $A_{\alpha}$ values drop monotonically. This latter behavior can be explained by the assumption that the $\alpha$-like Sn phase of $10 \AA$ maximum thickness exists exclusively at the $\mathrm{Sn} / \mathrm{Si}$ interface, and that the part of the Sn film in excess of $10 \AA$ 


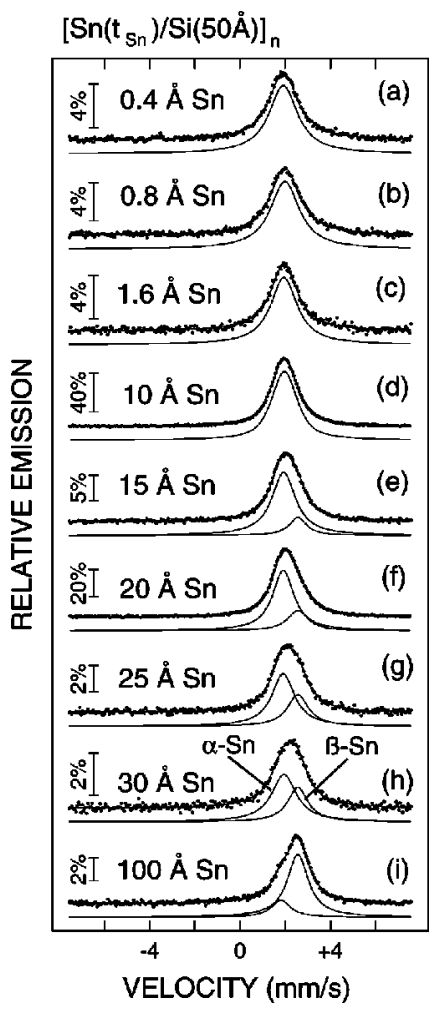

FIG. 3. Mössbauer (CEM) spectra at RT of $\left[\operatorname{Sn}\left(t_{\mathrm{Sn}}\right) / \operatorname{Si}(50 \AA)\right]_{n}$ multilayers with $t_{\mathrm{Sn}}=0.4 \AA$ (a), $0.8 \AA$ (b), $1.6 \AA$ (c), $10 \AA$ (d), 15 $\AA$ (e), $20 \AA$ (f), $25 \AA$ (g), $30 \AA$ (h), and $100 \AA$ (i) . The deposition temperatures were approximately RT. Least-squares fitting was performed with a single Lorentzian line for $\alpha$-like $\operatorname{Sn}\left(t_{\mathrm{Sn}} \leqslant 10 \AA\right)$ or with two single Lorentzian lines for the $\alpha$-like Sn and $\beta$-Sn, respectively $\left(t_{\mathrm{Sn}}>10 \AA\right)$.

thickness consists only of $\beta$-Sn. For this purpose we recall that in the thin absorber approximation ${ }^{30}$ the absolute experimental Mössbauer spectral area $A_{\alpha}^{a b s} \propto f_{\alpha} n_{\alpha} t_{\alpha}$ for $\alpha$-like Sn, and $A_{\beta}^{a b s} \propto f_{\beta} n_{\beta} t_{\beta}$ for $\beta$-Sn. Here $f_{\alpha}$ is the Lamb-Mössbauer factor ( $f$ factor), $n_{\alpha}$ is the number density of ${ }^{119} \mathrm{Sn}$ nuclei $\left({ }^{119} \mathrm{Sn}\right.$ nuclei $\left./ \mathrm{cm}^{3}\right)$, and $t_{\alpha}$ is the physical thickness $(\mathrm{cm})$ of the $\alpha-{ }^{119} \mathrm{Sn}$ layer. (For the $\beta$-Sn layer the corresponding quantities carry the index $\beta$.) The relative spectral area $A_{\alpha}$ normalized to the total spectral area, $\left(A_{\alpha}^{a b s}+A_{\beta}^{a b s}\right)$ is given by

$$
A_{\alpha}=\frac{f_{\alpha} n_{\alpha} t_{\alpha}}{\left(f_{\alpha} n_{\alpha} t_{\alpha}+f_{\beta} n_{\beta} t_{\beta}\right)} .
$$

In Fig. 4, values of $A_{\alpha}$, calculated according to Eq. (1), are shown (solid line), where we have used the values $t_{\alpha}$ $=10 \AA, t_{\beta}=t_{\mathrm{Sn}}-t_{\alpha}$, and $f_{\beta}=0.042$ (Ref. 31) and $f_{\alpha}$ $=0.21$ (see Sec. IV B). The comparison of experimental and calculated $A_{\alpha}$ values indicates a fair agreement. The deviation observed in Fig. 4 for $t_{\mathrm{Sn}}=50 \AA$ and $100 \AA$ is due to the fact that these data points were obtained from $\operatorname{Sn}(50 \AA)$ / $\operatorname{Si}(50 \AA)$ and $\operatorname{Sn}(100 \AA) / \operatorname{Si}(50 \AA)$ bilayers, where the rather thick $\beta$-Sn layer is closer to the surface than the $\alpha$-like Sn layer. Since the probability of escape of conversion electrons and Auger electrons decays as function of depth, the Möss- bauer signal $A_{\beta}^{a b s}$ will be enhanced relative to $A_{\alpha}^{a b s}$ in these bilayers. Then, this leads to an observed drop of the experimental data points relative to the calculated values. Figure 4 provides a strong evidence for our assumption of a layered $\beta$-Sn/ $\alpha$-like $\mathrm{Sn}$ structure.

Changing the Si thickness from $t_{\mathrm{Si}}=50 \AA$ to $20 \AA$ has no influence on the Sn phase formation. This has been checked by comparing spectra of RT grown $[\operatorname{Sn}(10 \AA) / \operatorname{Si}(50 \AA)]_{46}$ and $[\operatorname{Sn}(10 \AA) / \operatorname{Si}(20 \AA)]_{50}$ multilayers with identical $\mathrm{Sn}$ thicknesses. For $t_{\mathrm{Si}}=20 \AA$, the single line spectrum (not shown) was found to be similar to that in Fig. 3(d) (but slight broader), and pure $\alpha$-like-Sn is identified by the isomer shift value of $+2.011 \pm 0.004 \mathrm{~mm} / \mathrm{s}$ (compare with Table I).

The interesting aspect of the $\alpha$-like Sn phase grown in these multilayers is that it can be stabilized on $a$-Si by RT deposition up to $10 \AA$. We have also carried out lowtemperature depositions (LT, $T_{s}=-50{ }^{\circ} \mathrm{C}$ ) for $\mathrm{Sn}$ thicknesses of $1.6 \AA$ and $50 \AA$ in $\left[\operatorname{Sn}\left(t_{\mathrm{Sn}}\right) / \operatorname{Si}(50 \AA)\right]_{n}$ multilayers, with $n=20$ and 1 , respectively. The CEMS spectra (not shown) are very similar to the case of RT growth. $1.6 \AA \mathrm{Sn}$ grows as $\alpha$-like $\mathrm{Sn}$. LT deposition does not allow $\mathrm{Sn}$ to remain in its $\alpha$-like phase for 50- $\AA$-thick layers, except at the interface. Table I(c) displays the RT-Mössbauer parameters of these LT deposited multilayers. The relative spectral area of $\beta$-Sn in the $50 \AA \mathrm{Sn}$ sample $(62 \pm 10 \%)$ is much larger than that of $\alpha$-like $\operatorname{Sn}(38 \pm 10 \%)$. The experimental point corresponding to the LT growth of the $50-\AA \mathrm{Sn} / \mathrm{Si}$ bilayer fits well into the behavior of RT grown samples, as shown in Fig. 4. We conclude that LT growth does not favor the formation of $\alpha$-like Sn interface layers thicker than $10 \AA$.

\section{Raman spectroscopy}

We have measured the first-order Raman scattering by phonons (or vibrational excitations) in $\mathrm{Sn} / \mathrm{Si}$ multilayers at RT.

Figure 5 displays intensity versus Raman shift curves of four different samples: (a) $\operatorname{Si}(70 \AA) / \operatorname{Sn}(1000 \AA) / \operatorname{Si}(111)$, (b) $\quad[\operatorname{Sn}(15 \AA) / \operatorname{Si}(50 \AA)]_{6} / \operatorname{Si}(111), \quad$ (c) $\quad[\operatorname{Sn}(10 \AA) /$ $\operatorname{Si}(50 \AA)]_{50} / \operatorname{Si}(111)$, and (d) $\operatorname{Pt}(20 \AA) / \alpha-\operatorname{Sn}(60 \AA) /$ $\mathrm{InSb}(001)$, all grown at RT. The spectrum in Fig. 5(a) shows a sharp peak at $523 \mathrm{~cm}^{-1}$ that originates from transverse optical (TO) phonons of the crystalline Si substrate. ${ }^{32,33}$ The $1000-\AA-S n$ layer in this sample exists in the crystalline (metallic) $\beta$-Sn phase (see Sec. III A) with bct structure that cannot be detected by means of Raman spectroscopy. The broad peaks observed in Figs. 5(a)-5(c) at around 476 and $144 \mathrm{~cm}^{-1}$ are those of the TO-like and transverse acoustical (TA)-like excitations, respectively, of amorphous $(a-) \mathrm{Si}$, indicating the amorphous structure of the deposited Si layers. ${ }^{33}$

Since the total amount of $\mathrm{Si}$ in samples (b) and (c) is much larger than that of Sn, it is very difficult to detect any contribution of $\alpha$-Sn (if present at all) in these spectra. Furthermore the Raman peak of the TO phonons in $\alpha$-Sn grown on InSb is found at $180 \mathrm{~cm}^{-1}$ [Fig. 5(d)], in agreement with Ref. 34. Since the broad $144-\mathrm{cm}^{-1}$ peak of $a-\mathrm{Si}$ is in the same Raman-shift region, it is not possible to distinguish $\alpha$-Sn from $a$-Si because of considerable overlap of the spectra. We also note that if the $\alpha$-Sn layers were in a highly 
TABLE I. (a) ${ }^{119} \mathrm{Sn}$ Mössbauer parameters (isomer shift $\delta$, linewidth $\Gamma$ (for the Lorentzian line), and Gaussian width $\sigma$ (for the Voigt profile) of the $\left[\operatorname{Sn}\left(t_{\mathrm{Sn}}\right) / \operatorname{Si}(50 \AA)\right]_{n}$ multilayers measured at RT. All samples were deposited at RT. For $15 \AA \leqslant t_{\mathrm{Sn}} \leqslant 30 \AA$ the isomer shift of the $\beta$-Sn line was a fixed parameter at +2.56 $\mathrm{mm} / \mathrm{s}$, which is the literature value for bulk $\beta$-Sn (Ref. 24). (b) Mössbauer parameters (isomer shift $\delta$ and linewidth $\Gamma$ ) of $\alpha$-Sn in $500 \AA$ epitaxial ${ }^{119} \mathrm{Sn}$ on $\mathrm{InSb}(001)$ and of a $\beta$-Sn foil, both measured at RT, and comparison with the crystalline bulk values taken from the literature. ${ }^{23,24}$ (c) Mössbauer parameters of $\left[\operatorname{Sn}\left(t_{\mathrm{Sn}}\right) / \mathrm{Si}(50 \AA)\right]_{n}$ multilayers deposited at $-50{ }^{\circ} \mathrm{C}$ and measured at RT.

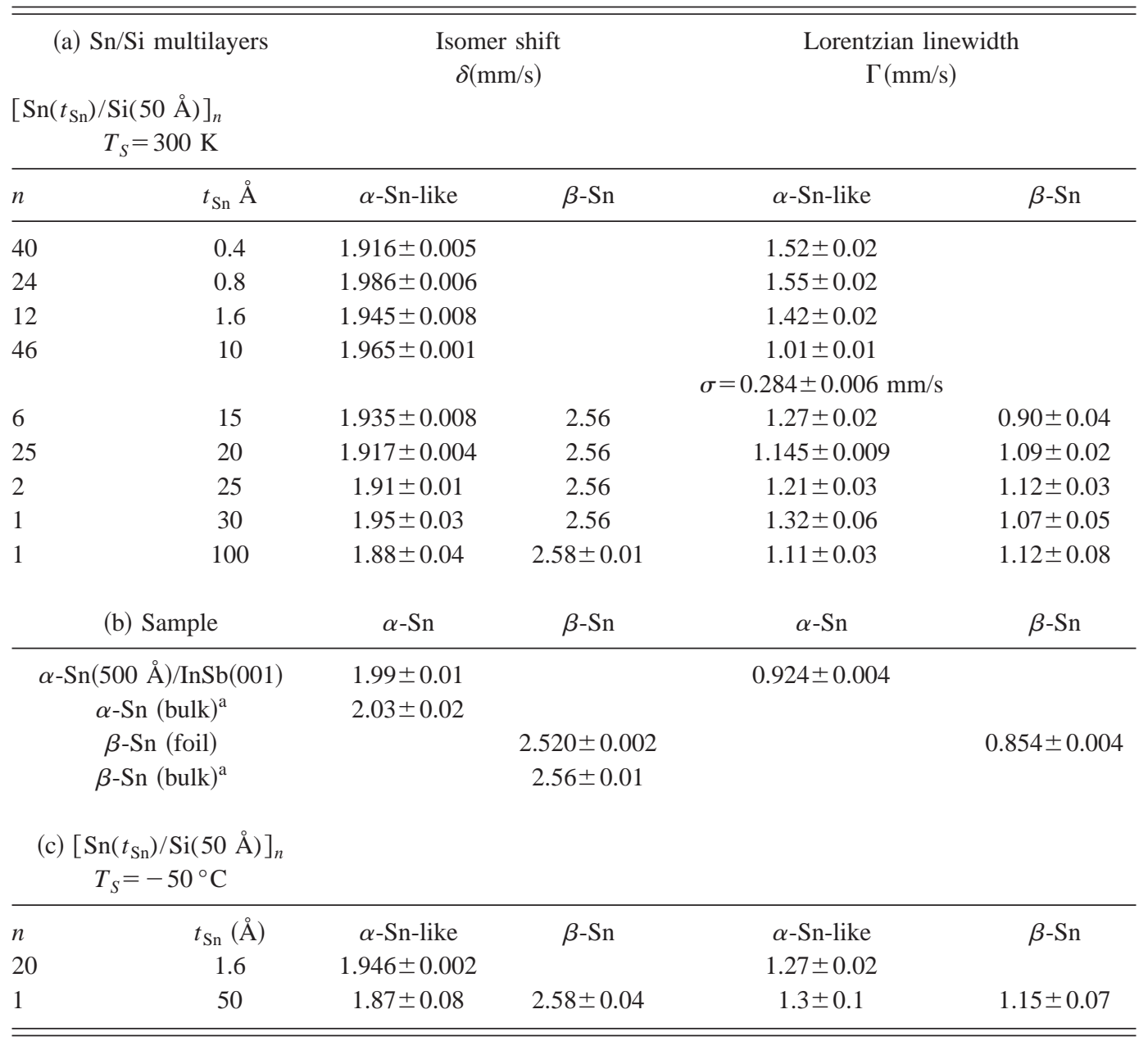

${ }^{\mathrm{a} S e e}$ Refs. 23 and 24.

disordered or amorphous $\alpha$-Sn-like structure, they are expected to show a broadened TO-like peak with reduced intensity, making its detection even more difficult.

\section{Nuclear resonant inelastic $x$-ray scattering}

NRIXS spectra were measured at RT on the following samples: ${ }^{119} \mathrm{Sn}(500 \AA) / \operatorname{InSb}(001), \quad\left[{ }^{119} \mathrm{Sn}(10 \AA) /\right.$ $\mathrm{Si}(20 \AA)]_{50}, \quad\left[{ }^{119} \operatorname{Sn}(10 \AA) / \operatorname{Si}(50 \AA)\right]_{46} \quad$ and $\left[{ }^{119} \operatorname{Sn}(20 \AA) / \operatorname{Si}(50 \AA)\right]_{25}$. The spectra (raw data) are shown in Figs. 6(a), 7(a), and 8(a), respectively. Since the spectra of the $\quad\left[{ }^{119} \operatorname{Sn}(10 \AA) / \operatorname{Si}(20 \AA)\right]_{50}$ and $\left[{ }^{119} \operatorname{Sn}(10 \AA) / \operatorname{Si}(50 \AA)\right]_{46}$ multilayers were found to be identical within statistical error bars, they were added in order to improve the statistical quality; this result is displayed in Fig. 7(a).

The main features of these NRIXS spectra are as follows: an elastic central peak at $E-E_{0}=0 \mathrm{meV}$ and side bands at lower and higher energy. Here, $E$ is the incident photon en- ergy and $E_{0}=23.88 \mathrm{keV}$ is the nuclear-resonance energy. The elastic peak dominates the spectrum as expected for solids with reasonable probability for recoilless absorption (reasonably high Lamb-Mössbauer factor or $f$ factor). Photons with less energy than $E_{0}$ can excite the nuclear resonance by annihilation of a phonon (low-energy side band). The highenergy side band corresponds to the phonon creation. Phonon annihilation is proportional to the temperaturedependent phonon occupation number, whereas the creation of phonons can also occur spontaneously, which explains the observed asymmetry in the spectra.

Figure 6(a) displays the NRIXS spectrum of a thick (500 A) $\alpha$-Sn film. The result obtained looks similar to that measured in Ref. 35 on $\operatorname{Sn}(2000 \AA) / C d T e(001)$, where the crystalline $\alpha$-Sn phase was observed, too. The instrumental resolution function of $0.8 \mathrm{meV}$ width is plotted around $E-E_{0}$ $=0 \mathrm{meV}$. Especially sharp phonon excitation peaks are observed at 6, 11, and $22 \mathrm{meV}$ (phonon creation part of the 


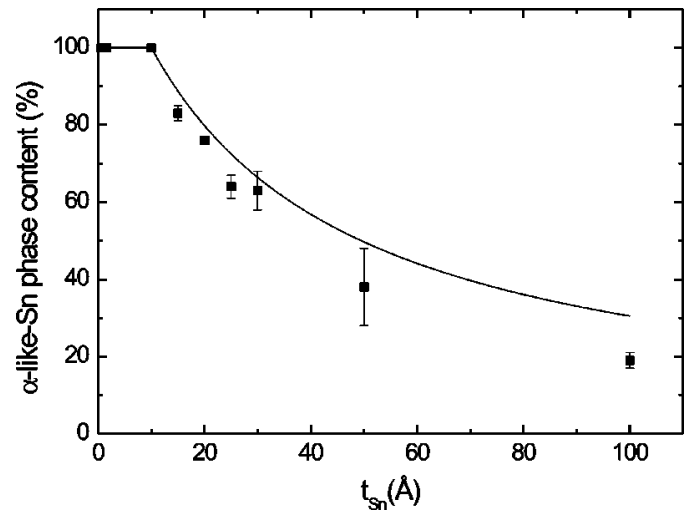

FIG. 4. Relative $\alpha$-like-Sn content in the Sn layers as a function of Sn layer thickness, $t_{\mathrm{Sn}}$. The samples are $\left[\operatorname{Sn}\left(t_{\mathrm{Sn}}\right) / \operatorname{Si}(50 \AA)\right]_{n}$ multilayers for $t_{\mathrm{Sn}} \leqslant 30 \AA$, and $[\operatorname{Sn}(50 \AA) / \mathrm{Si}(50 \AA)]_{1}$ and $[\operatorname{Sn}(100 \AA) / \operatorname{Si}(50 \AA)]_{1}$ bilayers for $t_{\mathrm{Sn}}>30 \AA$. The bilayer with $t_{\mathrm{Sn}}=50 \AA$ was grown at $T_{s}=-50^{\circ} \mathrm{C}$, and all other samples were grown at RT. The experimental data points correspond to the relative Mössbauer spectral areas, $A_{\alpha}$, of $\alpha$-like $\mathrm{Sn}$ (full squares). They are compared with calculations using Eq. (1) and the values of the Lamb-Mössbauer factors $f_{\beta}=0.042$ (Ref. 31) and $f_{\alpha}=0.21$ (solid line), with $f_{\alpha}$ obtained from NRIXS measurements.

spectrum), indicating the crystallographic long-range order present in this sample.

Figure 6(b) displays the phonon-excitation-probability function, obtained directly from the experimental data after normalization and subtraction of the elastic peak contribution as described in Refs. 19, 36-38. This spectrum can be decomposed into single and multiphonon contributions. At ambient temperature one may recognize various contributions to this spectrum, corresponding to inelastic nuclear absorption accompanied by excitation or annihilation of different number of phonons. The normalized probability of the inelastic nuclear absorption, $W(E)$, can be decomposed in terms of a multiphonon expansion ${ }^{19,37}$

$$
W(E)=\left[\delta(E)+\sum_{n=1}^{\infty} S_{n}(E)\right] f
$$

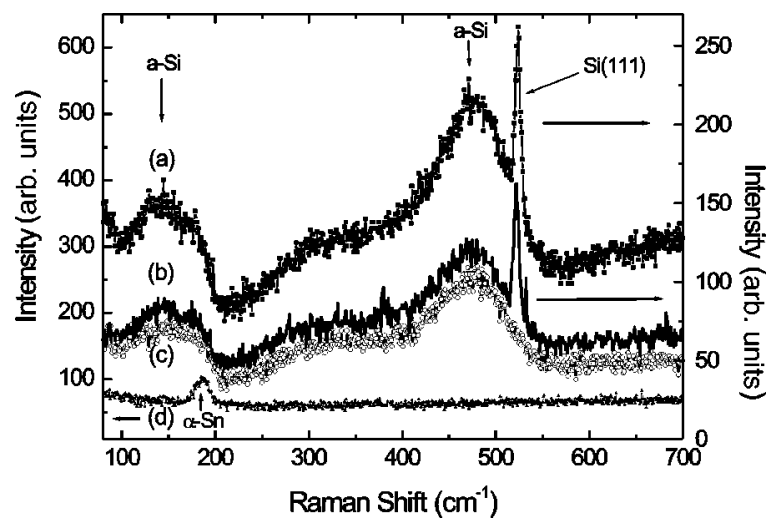

FIG. 5. Raman spectra measured at RT in He atmosphere of (a) $\operatorname{Si}(70 \AA) / \operatorname{Sn}(1000 \AA) / \operatorname{Si}(111) ; \quad$ (b) $\quad[\operatorname{Sn}(15 \AA) / \operatorname{Si}(50 \AA)]_{6} /$ $\mathrm{Si}(111) ; \quad$ (c) $\quad[\operatorname{Sn}(10 \AA) / \operatorname{Si}(50 \AA)]_{50} / \operatorname{Si}(111) ; \quad$ and $\quad(d)$ $\operatorname{Pt}(20 \AA) / \operatorname{Sn}(60 \AA) / \operatorname{InSb}(001)$.
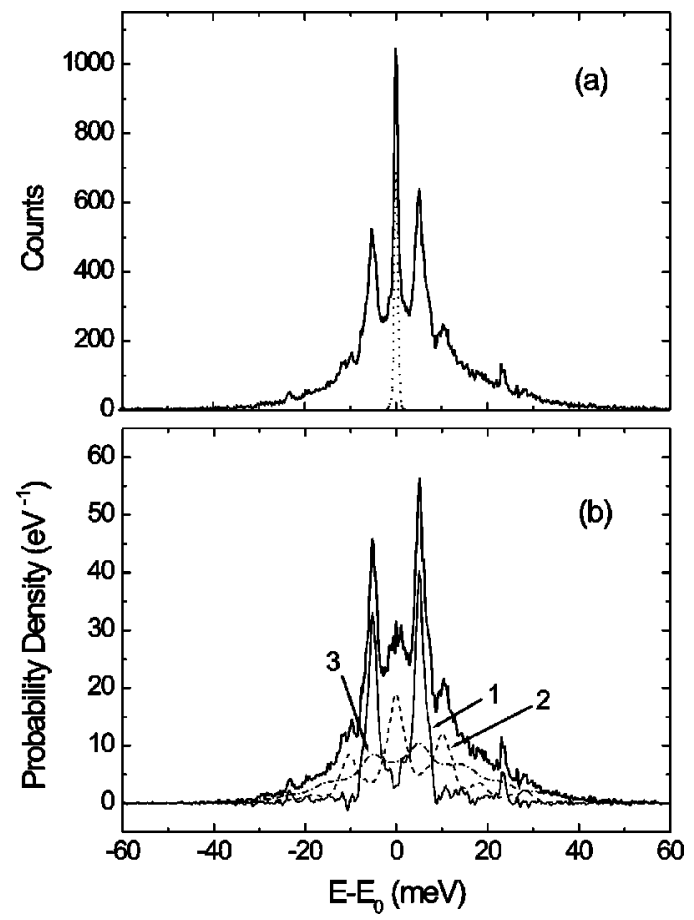

FIG. 6. (a) NRIXS spectrum (raw data) of 500- -thick epitaxial $\alpha-{ }^{119} \mathrm{Sn}$ on InSb(001) (solid line) measured at RT . The dotted line shows the instrumental resolution function; (b) decomposition of the NRIXS spectrum (after normalization and removal of the elastic peak) in a one-phonon (1), two-phonon (2), and higher-order phonon (3) contribution.

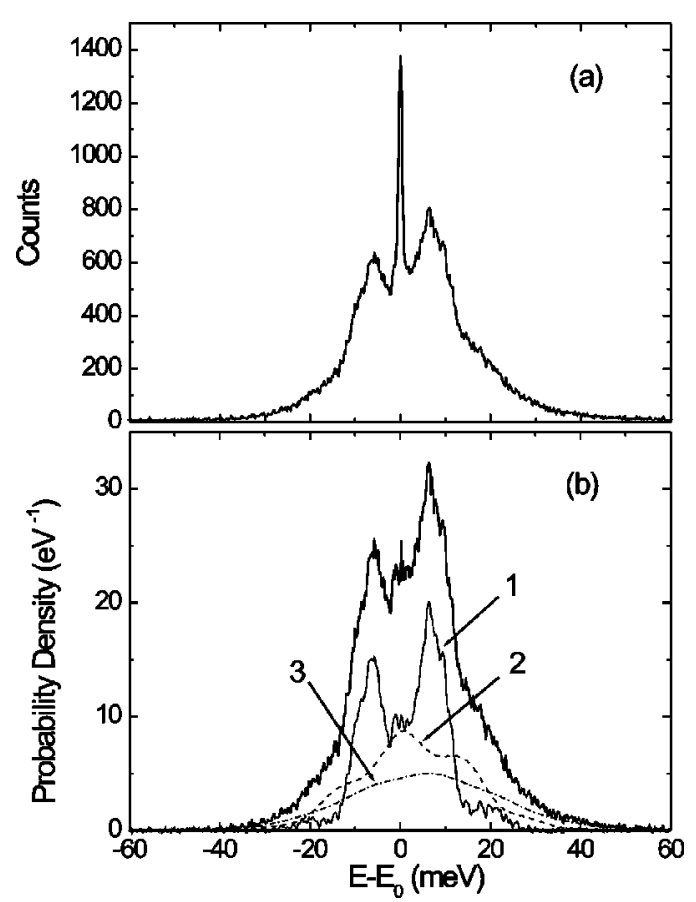

FIG. 7. (a) Sum of NRIXS spectra (raw data) of $\left[{ }^{119} \mathrm{Sn}(10 \AA) / \mathrm{Si}(50 \AA)\right]_{50}$ and $\left[{ }^{119} \operatorname{Sn}(10 \AA) / \mathrm{Si}(20 \AA)\right]_{46}$ measured at RT; (b) decomposition of the NRIXS spectrum (after normalization and removal of the elastic peak) in a one-phonon (1), a twophonon (2), and a higher-order phonon (3) contribution. 

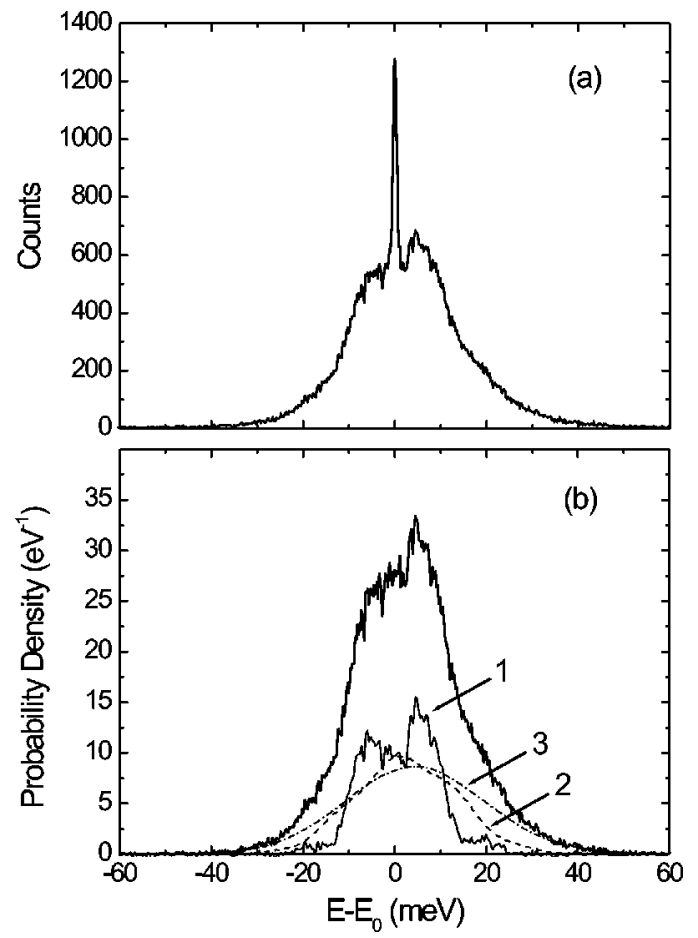

FIG. 8. (a) NRIXS spectrum (raw data) of $\left[{ }^{119} \operatorname{Sn}(20 \AA) / \operatorname{Si}(50 \AA)\right]_{25}$ measured at RT; (b) decomposition of the NRIXS spectrum (after normalization and removal of the elastic peak) in a one-phonon (1), a two-phonon (2), and a higher-order phonon (3) contribution.

Here the $\delta$ function describes the elastic part of the nuclear absorption (zero-phonon term), and the $n$th term of the series $S_{n}(E)$ represents the inelastic nuclear absorption accompanied by creation (annihilation) of $n$ phonons. $f$ is the Lamb-Mössbauer factor. The one-phonon term is given by

$$
S_{1}(E)=\frac{E_{R} g(E)}{E\left(1-e^{-\beta E}\right)}
$$

and the subsequent terms in the harmonic approximation may be found through the recursive relation

$$
S_{n}(E)=\frac{1}{n} \int_{-\infty}^{\infty} S_{1}\left(E^{\prime}\right) S_{n-1}\left(E-E^{\prime}\right) d E^{\prime} .
$$

Here $\beta=\left(k_{B} T\right)^{-1}$ with $k_{B}$ the Boltzmann constant, $T$ is the temperature, $E_{R}=\hbar^{2} k^{2} / 2 M$ the recoil energy of the free nucleus, where $k$ the wave vector of the photon, and $M$ the mass of the atom. The function $g(E)$ is the normalized phonon (or vibrational) density of states (VDOS). The relative contribution $\int S_{n}(E) d E$ of the $n$-phonon term is given by $(-\ln f)^{n} / n !$.

In order to extract the phonon-excitation probabilities, the program PHOENIX (Ref. 36) was used. The contributions of the one-, two-, and higher-order phonon-excitation probabilities are displayed in Figs. 6(b), 7(b), and 8(b), respectively. The clear distinction of the multiphonon contributions is no longer possible, if the Lamb-Mössbauer factor is small as in $\beta$-Sn. ${ }^{31,35}$ In this case, the expansion in Eq. (2) converges slowly, and a large number of terms has to be taken into account. With an $f$ factor of $0.16,{ }^{35} \alpha$-Sn has significant multiphonon contributions, but not to the degree of preventing the separation of $S_{1}(E), S_{2}(E), S_{3}(E)$.

The phonon (or vibrational) density of states $g(E)$ in Eq. (3) is proportional to the one-phonon term in the expansion of Eq. (2). We deconvoluted the measured spectra of Figs. 6(b), 7(b), and 8(b) with the resolution function and applied Eq. (3) to obtain the VDOS. Again the program PHOENIX (Ref. 36) was used for that purpose. The results are shown in Figs. 9(a)-9(c), respectively.

In the case of the 500- $\AA$-thick epitaxial $\alpha$-Sn film [Fig. 9(a)], $g(E)$ shows several distinct peaks at positions that are in very good agreement over the whole energy range with those theoretically predicted in Ref. 6 for bulk $\alpha$-Sn, although in our case the features are not as sharp as in the calculation [bold solid line in Fig. 9(a)]. We observe the TA peak at $5 \mathrm{meV}$, the longitudinal acoustical (LA) peak at 15 $\mathrm{meV}$, the longitudinal optical (LO) peak at $20 \mathrm{meV}$, and the strong TO peak at $23 \mathrm{meV}$. There is also a pronounced shoulder near $7 \mathrm{meV}$ in the TA band.

The NRIXS spectrum of the ${ }^{119} \operatorname{Sn}(10 \AA) / \mathrm{Si}$ multilayer, as displayed in Fig. 7(a), looks quite different from the one plotted in Fig. 6(a) for the crystalline $\alpha$-Sn film. As compared to $\alpha$-Sn, the ${ }^{119} \mathrm{Sn}(10 \AA) / \mathrm{Si}$ multilayer exhibits broader phonon creation and annihilation features and disappearance of the sharp peak at 11 and $22 \mathrm{meV}$. This indicates the existence of considerable atomic disorder in the $10 \AA$ interfacial Sn layer. For example, a similar disappearance of sharp peaks has been observed in NRIXS spectra of thin films of amorphous $\mathrm{TbFe}_{2}$ films, as compared to films of the crystalline Laves phase $\mathrm{TbFe}_{2} \cdot{ }^{20}$ Comparing Figs. 6(a) and 7(a), and taking into account that for both cases we measured almost the same isomer shift (of $\alpha$-like Sn) with CEMS, we presume that the 10 - $\AA$-thick $\mathrm{Sn}$ layers in $\mathrm{Sn} / a$-Si multilayers exist in a long-range disordered, amorphous $\alpha$-like structure. Figure 7(b) shows the decomposition of the NRIXS spectrum of Fig. 7(a), after normalization and removal of the elastic peak in one-, two-, and higher-order-phonon contributions. The VDOS of the 10- $\AA \mathrm{Sn} / a$-Si multilayer [Fig. 9(b)] looks strikingly different from the DOS of the crystalline $\alpha$-Sn film [Fig. 9(a)]. The vibrational spectrum of the $\operatorname{Sn}(10 \AA$ ) $/ a$-Si multilayer is characterized by two extremely broad peaks positioned at $10 \mathrm{meV}$ and about 20-22 meV, respectively. The dominant $10-\mathrm{meV}$ peak also shows a shoulder near $7 \mathrm{meV}$. On the high-energy side the broad band with a maximum near $\sim 22 \mathrm{meV}$ extends up to a cutoff energy of about $28 \mathrm{meV}$, which is much larger than the position (23 $\mathrm{meV}$ ) of the TO peak of crystalline $\alpha$-Sn [Fig. 9(a)]. It is obvious that the four rather sharp phonon peaks of crystalline $\alpha$-Sn (in particular its dominant TO peak and the TA band) have been drastically modified with respect to energy and intensity in the 10- $\AA$-thick amorphous $\alpha$-like Sn interface layers.

Figure 8(a) shows the NRIXS spectrum of the $\left[{ }^{119} \operatorname{Sn}(20 \AA) / \operatorname{Si}(50 \AA)\right]_{25}$ multilayer. From CEMS we know that $20 \AA \mathrm{Sn}$ in these multilayers consists of $10-\AA$-thick interfacial $\alpha$-like Sn and $\sim 10 \AA \beta$-Sn. Since we concluded from Figs. 7(a) and 7(b) that $10 \AA \mathrm{Sn}$ at the $\mathrm{Sn} / \mathrm{Si}$ interface is 

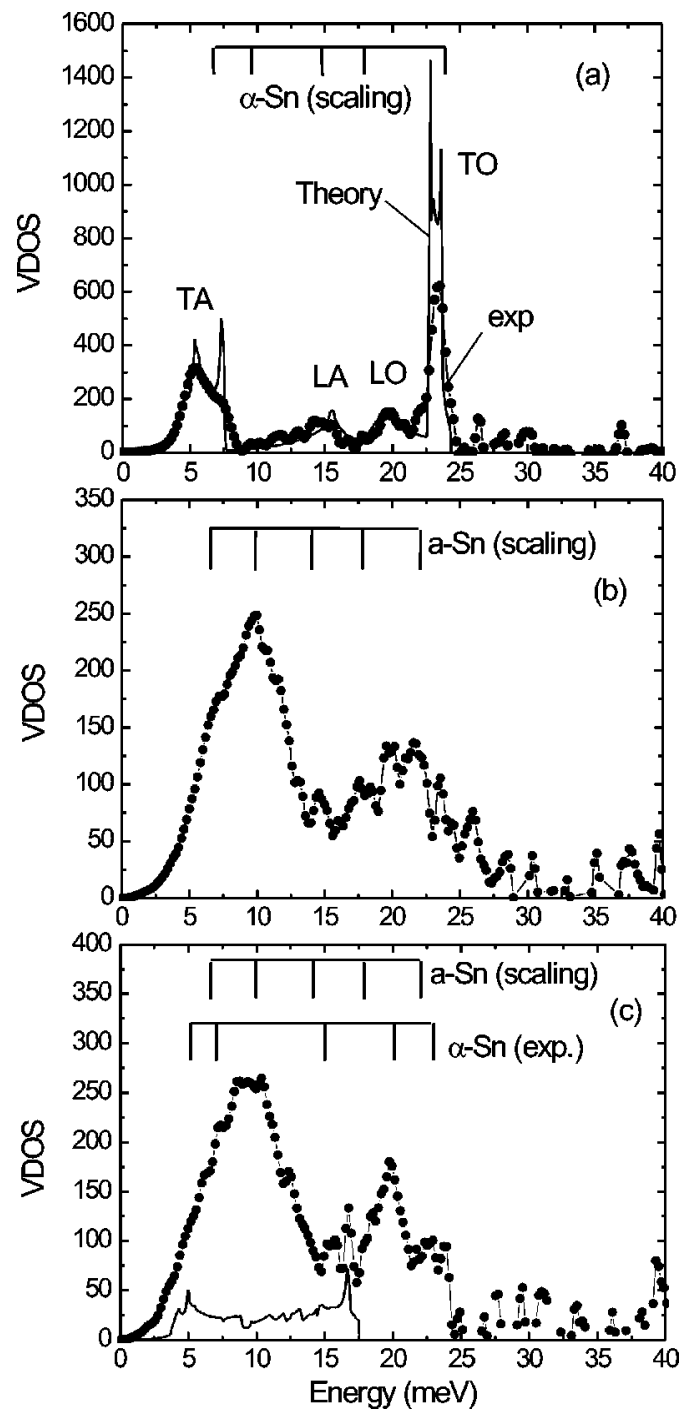

FIG. 9. Vibrational density of states (VDOS): (a) for $\alpha-{ }^{119} \operatorname{Sn}(500 \AA) / \operatorname{InSb}(001)$, derived from Fig. 6(b) (full circles); the theoretical phonon DOS for bulk $\alpha$-Sn is also shown for comparison (bold solid line) (Ref. 6). (The original theoretical curve was convoluted with our instrumental resolution function.) (b) For $\left[{ }^{119} \operatorname{Sn}(10 \AA) / a-\operatorname{Si}(50 \AA)\right]_{46}$ and $\left[{ }^{119} \operatorname{Sn}(10 \AA) / a-\operatorname{Si}(20 \AA)\right]_{50}$ multilayers, derived from Fig. 7(b) (full circles). (c) For $\left[{ }^{119} \operatorname{Sn}(20 \AA) / a-\operatorname{Si}(50 \AA)\right]_{25}$ multilayer, derived from Fig. 8(b) (full circles); the bar diagram $[\alpha-\operatorname{Sn}(\exp )]$ indicates the measured peak and shoulder positions for $\alpha{ }^{119}$ Sn $(500 \AA)$ in (a); the theoretical VDOS for bulk $\beta$-Sn (in arbitrary units) is also shown for comparison (bold solid line) (Ref. 6). The other bar diagrams indicate the energies of the prominent peak or shoulder of the various phononlike bands predicted for amorphous- $\alpha$-like Sn $(a$-Sn) by scaling [use of Eq. (5), see also Fig. 10].

in an amorphous state, the spectrum displayed in Fig. 8(a) must be a superposition of two contributions: one originating from amorphous interfacial $\alpha$-like $\mathrm{Sn}$, and one from crystalline $\beta$-Sn. Since in Fig. 8(a) the elastic peak does not completely disappear [as would occur for pure $\beta$-Sn at $300 \mathrm{~K}$ (Refs. 31 and 35)], we conclude that the $\operatorname{Sn}(20 \AA) / a-\mathrm{Si}$ multilayer contains amorphous- $\alpha$-like $\mathrm{Sn}$ and additionally a contribution of $\beta$-Sn, in agreement with the CEMS results.
TABLE II. Recoilless fraction (Lamb-Mössbauer factor $f$ ), mean kinetic energy per atom $\bar{T}$, and mean atomic force constant $\bar{K}$ for the Sn layers, derived from NRIXS measurements.

\begin{tabular}{lccc}
\hline \hline Sample & $f$ & $\begin{array}{c}\bar{T} \\
(\mathrm{meV})\end{array}$ & $\begin{array}{c}\bar{K} \\
(\mathrm{~N} / \mathrm{m})\end{array}$ \\
\hline$\alpha$-Sn(500 $\mathrm{A}) / \mathrm{InSb}(001)$ & $0.14 \pm 0.02$ & $13.9 \pm 0.3$ & $117 \pm 33$ \\
$\alpha$-Sn(2000 $) / \mathrm{CdTe}(001){ }^{\text {a }}$ & $0.16 \pm 0.02$ & $13.4 \pm 0.2$ & $155 \pm 16$ \\
Amorphous $\alpha$-like-Sn in & & & \\
{$[\mathrm{Sn}(10 \AA) / \mathrm{Si}(20 \AA)]_{50}+$} & $0.21 \pm 0.01$ & $13.5 \pm 0.2$ & $168 \pm 18$ \\
{$[\mathrm{Sn}(10 \AA) / \mathrm{Si}(50 \AA)]_{46}$} & & & \\
$\mathrm{Amorphous} \alpha$-like-Sn $+\beta$-Sn & & & \\
{$[\mathrm{Sn}(20 \AA) / \mathrm{Si}(50 \AA)]_{25}$} & $0.13 \pm 0.02$ & $13.0 \pm 0.2$ & $97 \pm 20$ \\
${ }^{119} \mathrm{Sn}$ ion-implanted & $0.133 \pm 0.006$ & & \\
in (bulk) $\alpha$-Sn ${ }^{\mathrm{b}}$ & & & \\
\hline
\end{tabular}

${ }^{\text {a See Ref. } 35 .}$

${ }^{\mathrm{b}}$ See Ref. 4.

This sample also presents a dominant multiphonon contribution [Fig. 8(b)], similar to the $\beta$-Sn case. ${ }^{31,35}$ As observed for 10- $\AA$ amorphous $\alpha$-like Sn in Fig. 9(b), the VDOS shown in Fig. 9(c) also exhibits broad features. In particular, the width of the lower-energy peak in Fig. 9(c) near $\sim 10 \mathrm{meV}$ appears to be larger than that of the corresponding peak in Fig. 9 (b). One reason for this broadening is the additional contribution of $\beta$-Sn in the VDOS of Fig. 9(c), which is known to extend only up to $\sim 17 \mathrm{meV}\left(\sim 140 \mathrm{~cm}^{-1}\right)$ (Refs. 6 and 31) [see solid curve in Fig. 9(c)], and thus essentially overlaps with the $10-\mathrm{meV}$ VDOS peak of amorphous $\alpha$-like Sn. Similar to the case of Fig. 9(b), the strong TO peak of crystalline $\alpha$-Sn near $23 \mathrm{meV}$ appears to be largely suppressed also in the VDOS of $\beta$-Sn covered interfacial amorphous- $\alpha$-like Sn [Fig. 9(c)]. The peak near $20 \mathrm{meV}$ (which coincides with the position of the LO peak of crystalline $\alpha$-Sn) is more pronounced in Fig. 9(c) than in Fig. 9(b).

From the normalized probability of inelastic nuclear absorption, $W(E)$, the Lamb-Mössbauer recoilless factor $f$, the mean kinetic energy per atom $\bar{T}$, and the mean atomic force constant $\bar{K}$ have been obtained after removal of the elastic contribution, by using model-independent sum rules. ${ }^{39}$ Moreover, the vibrational density of states $g(E)$, provides information on the vibrational entropy per atom $S .{ }^{37}$ Table II displays values of $f, \bar{T}$, and $\bar{K}$ thus obtained. The value of the $f$ factor obtained for $500-\AA \alpha-\operatorname{Sn}$ on $\operatorname{InSb}(001), f=0.14$ \pm 0.02 , is very close to the bulk value of $\alpha$-Sn given in Ref. $35(f=0.16 \pm 0.02)$ for $2000 \AA$ epitaxial $\alpha$-Sn grown on $\mathrm{CdTe}(001)$. For the case of the amorphous- $\alpha$-like-Sn sample $\left\{[\operatorname{Sn}(10 \AA) / \operatorname{Si}(20,50 \AA)]_{50}\right\}$, the $f$ factor of $0.21 \pm 0.01$ is found to be significantly larger than these values. The sample that contained amorphous $\alpha$-like $\mathrm{Sn}$ and $\beta$-Sn $\left\{[\operatorname{Sn}(20 \AA) / \operatorname{Si}(50 \AA)]_{25}\right\}$ shows a much smaller $f$ factor, $f$ $=0.13 \pm 0.02$, suggesting that this value is about the average value between $f=0.21$ for interfacial amorphous- $\alpha$-like Sn and $f=0.042$ for crystalline $\beta$-Sn. The latter sample has also the smallest average kinetic energy $\bar{T}$ of all samples (Table II). 


\section{DISCUSSION}

\section{A. Structure}

The Raman spectra [Figs. 5(a)-5(c)] demonstrate conclusively that the evaporated $\mathrm{Si}$ layers in the $\mathrm{Sn} / \mathrm{Si}$ multilayers are amorphous. This result agrees with the wide-angle XRD scans [Figs. 2(a)-2(c)], where no sharp Bragg peaks of crystalline $\mathrm{Si}$, except that of the $\mathrm{Si}(111)$ substrate, are observed. (This conclusion is based on the hypothesis that the RTdeposited Si layers are polycrystalline.)

Concerning the Sn phases in the multilayers, Bragg reflections of crystalline $\alpha$-Sn have not been detected on any of our $\mathrm{Sn} / a$-Si multilayers, for instance in Fig. 2(a) for $t_{\mathrm{Sn}}$ $=10 \AA$ and Fig. 2(b) for $t_{\mathrm{Sn}}=20 \AA$. On the other hand, the crystalline $\beta$-Sn (400) Bragg reflection is observed in Fig. 2(b) for the multilayer with $t_{\mathrm{Sn}}=20 \AA$, which (according to the CEMS result, Fig. 4) contains much more $\alpha$-like Sn (70\%) than $\beta$-Sn $(30 \%)$. This demonstrates that the XRD sensitivity is high enough to detect the crystalline $\alpha$-Sn phase (if any was present) in multilayers with $t_{\mathrm{Sn}}=10 \AA$ or $t_{\mathrm{Sn}}=20 \AA$. For this reason, and since Bragg reflections of crystalline $\alpha$-Sn have not been observed for $t_{\mathrm{Sn}}=10-20 \AA$ (or any other $\mathrm{Sn}$ thicknesses), we may conclude that the $\alpha$-Sn-like phase [which is unambiguously identified by CEMS (Fig. 3) in such multilayers] has a disordered (amorphous) structure that does not yield Bragg peaks.

The CEMS result displayed in Fig. 4 demonstrates that the amorphous- $\alpha$-like-Sn layer of $\sim 10 \AA$ thickness is located at the interface with amorphous Si. Moreover we like to emphasize that the isomer-shift (i.e., chemical-shift) values, $\delta$, of interfacial amorphous- $\alpha$-like Sn (Table I) are in very good agreement with $\delta$ values of pure-crystalline epitaxial $\alpha$-Sn films and of bulk $\alpha$-Sn (Table I). This observation proves that interfacial $\mathrm{Sn}-\mathrm{Si}$ alloy formation does not occur in the multilayers, as expected from the thermodynamic phase diagram. ${ }^{17}$ This is corroborated by the fact that the ${ }^{119} \mathrm{Sn}$ isomer shift of $\alpha$-Sn was found to be remarkably larger (by $0.26 \mathrm{~mm} / \mathrm{s}$ ) than that of epitaxial metastable $\mathrm{Si}_{1-x} \mathrm{Sn}_{x}$ dilute-alloy films, ${ }^{40}$ i.e., the isomer shift (relative to pure $\alpha-\mathrm{Sn}$ ) is reduced by $\mathrm{Sn}-\mathrm{Si}$ alloying, which is not observed in our samples (Table I). Further, the similar isomer shifts suggest that the average local electronic structure (which is related to the average local environment or shortrange order) in the amorphous- $\alpha$-like-Sn interface is similar to that of crystalline $\alpha$-Sn, since the isomer shift is proportional to the $s$-electron density at the Mössbauer nucleus. ${ }^{41}$ We also can notice an enhancement of the Mössbauer linewidth of the amorphous- $\alpha$-like-Sn interfacial layer as compared to the linewidth of the $\beta$-Sn phase in the multilayers (Table I). This excess broadening may originate from a small distribution of isomer shifts and/or weak quadrupole interactions in the disordered (amorphous) $\alpha$-like-Sn phase. When comparing with literature reports, ${ }^{4}$ the isomer shift of amorphous- $\alpha$-like Sn produced by ${ }^{119} \mathrm{Sn}$-ion implantation in (bulk) $\alpha$-Sn $(2.14 \pm 0.04 \mathrm{~mm} / \mathrm{s})$ is slightly larger than that of bulk $\alpha$-Sn and that of our amorphous Sn (Table I). Differences in the deviations from the ideal local tetrahedral structure in both types of samples may account for this small difference in isomer shifts.

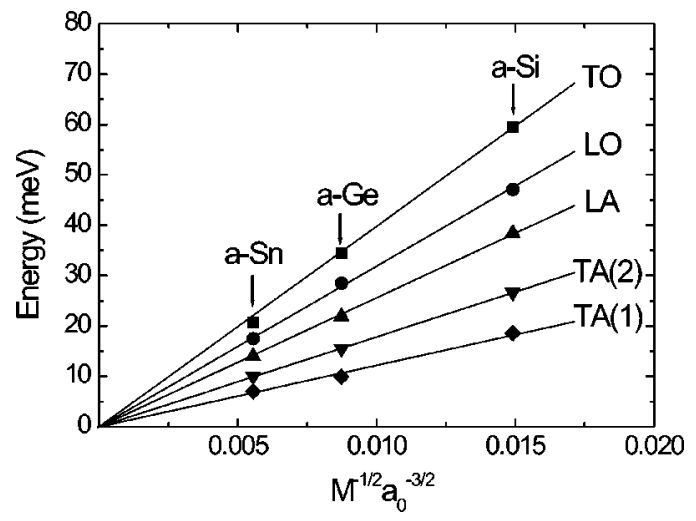

FIG. 10. Measured energies of the prominent peak or shoulder of various phononlike bands related to the VDOS of (bulklike) $a$-Ge (Ref. 33) and (bulklike) $a$-Si (Refs. 33 and 42) plotted versus $M^{-1 / 2} a_{0}^{-3 / 2}$ ( $M$ : atomic mass number; $a_{0}$ : lattice parameter). The straight lines are least-squares fits to the data points for $a$-Ge and $a$-Si and were forced to pass through the origin. The data for amorphous $\alpha$-like Sn ( $a$-Sn) obtained from Fig. 9(b) scale well with the data of $a$-Ge and $a$-Si. [The values $a_{0}(\mathrm{Si})=5.43 \AA, a_{0}(\mathrm{Ge})$ $=5.65 \AA$, and $a_{0}(\alpha-\mathrm{Sn})=6.489 \AA$ were used].

\section{B. Vibrational dynamics}

\section{Vibrational density of states}

One of our most important results is the observation of remarkable differences in the VDOS of the crystalline (epitaxial) $\alpha$-Sn film [Fig. 9(a)] and of 10- $\AA$-thick $\alpha$-like Sn in the Sn/a-Si multilayers [Fig. 9(b)]. The arguments for the amorphous structure of this interfacial $\alpha$-like-Sn (called amorphous $(a-) \mathrm{Sn}$ in the following) have been presented in Sec. IV A. Moreover, the energies of the prominent features (peaks and shoulders) in the VDOS bands [Fig. 9(b)] are typical for the amorphous phase of $\alpha$-Sn and are predicted by scaling of the respective energies of $a-\mathrm{Ge}$ and $a$-Si. This is demonstrated in Fig. 10, where we show the known energies of the prominent VDOS features (peaks and shoulders) of various phononlike bands (TO, LO, LA, and TA type) of amorphous ( $a$-) Ge (Ref. 33) and $a$-Si (Refs. 33 and 42) as a function of $M^{-1 / 2} a_{0}^{-3 / 2}$. ( $M$ is the atomic mass number and $a_{0}$ the lattice parameter). It is known that the prominent energies $E_{i}$ of a certain vibrational band $i$ of $a$-Ge and $a$-Si (and also of crystalline (cr-) Ge and $\mathrm{cr}-\mathrm{Si}$ ) are proportional to $M^{-1 / 2} a_{0}^{-3 / 2} \cdot 33,43,44$ This proportionality is well described by the straight lines in Fig. 10. The corresponding vibrational energies of $a$-Sn may be predicted by the scaling relation ${ }^{33}$

$$
\omega_{i}(\mathrm{Sn})=\omega_{i}(\mathrm{Ge}, \mathrm{Si})\left(\frac{M_{\mathrm{Sn}}}{M_{\mathrm{Ge}, \mathrm{Si}}}\right)^{-1 / 2}\left(\frac{a_{0}(\alpha-\mathrm{Sn})}{a_{0}(\mathrm{Ge}, \mathrm{Si})}\right)^{-3 / 2} .
$$

The results thus obtained are shown as a bar diagram for $\alpha$-Sn in Fig. 9(a) and for $a$-Sn in Figs. 9(b) and 9(c). For $\alpha$-Sn, Fig. 9(a), the differences between the energies predicted from scaling and the experimentally observed peak/ shoulder are small and at most $2 \mathrm{meV}$; best agreement is found for the TO mode. For interfacial $a$-Sn [Fig. 9(b)], the experimentally observed energies of prominent features and those predicted from scaling also are in good agreement; in 
particular, the observed dominant peak near $10 \mathrm{meV}$ of the TA-like band [called TA(2) in Fig. 10] and the shoulder near $7 \mathrm{meV}$ of the TA-like band [called TA(1) in Fig. 10] are well reproduced by scaling. In the region of the TO-like band the maximum at $\sim 20.6 \mathrm{meV}$ of the observed broad hump coincides within only $\sim 1.5 \mathrm{meV}$ with the position of the predicted TO-like peak [Fig. 9(b)]. We also find less intense peaks at 14 and $17.5 \mathrm{meV}$ in the experimental data that coincide with the LA-like and LO-like peak positions obtained from scaling. The larger scatter of the data points at and above $\sim 24 \mathrm{meV}$ [Fig. 9(b)] makes identification of peaks in that region uncertain. The good agreement between experimental and predicted (from scaling) vibrational energies can also be noticed in Fig. 10, where our experimental values for interfacial $a$-Sn all fall in the respective straight line [given by Eq. (5)]. This good agreement provides further strong evidence for the amorphous nature of the 10 - $\AA$-thick $\alpha$-like Sn layers embedded between $a$-Si layers.

It is worthwhile mentioning that the strong TA-like band [Fig. 9(b)] of interfacial $a$-Sn is shifted towards higher energy by $2-3 \mathrm{meV}$ relative to its crystalline bulklike counterpart [Fig. 9(a)]. Further, the intensity ratio TA(2)/TA(1) (10meV peak to $7-\mathrm{meV}$ peak) of interfacial $a-\mathrm{Sn}$ is $>1$, while its crystalline counterpart (7-meV shoulder to $5-\mathrm{meV}$ peak) is $<1$. This difference may be possibly the consequence of the quasi-two-dimensional nature of our 10-Å-thick $a$-Sn films. We speculate that interface coupling (hybridization) of TA-like modes of interfacial $a$-Sn with the TA-like modes of $a$-Si that are higher in energy [near 18.6 and $26.7 \mathrm{meV}$ (Ref. 33)] might cause these VDOS differences between (bulklike) $\alpha$-Sn and interfacial $a$-Sn.

Inspection of Figs. 9(a) and 9(b) reveals that the VDOS of interfacial $a$-Sn extends to about $11 \%$ higher energies than the VDOS of $\alpha$-Sn, and surprisingly the strong TO band of $\alpha$-Sn is largely suppressed in interfacial $a$-Sn. Both effects may be caused by the very high degree of local atomic disorder in $a$-Sn as compared to $\alpha$-Sn. As has been pointed out in Ref. 2 on Raman spectroscopy, the strength of the TA Raman band of $a-\mathrm{Si}$ is enhanced (and the strength of the TO Raman band is reduced) by increasing disorder. The disorder in $a$-Si may be enhanced, for instance, by increasing average bond angle fluctuations, resulting in larger Raman linewidths of the TO band ${ }^{45}$ combined with an enhanced ratio of the strengths of the TA to the TO Raman bands. ${ }^{46}$ As the Raman bands reflect the VDOS, we may expect similar behavior of the VDOS bands upon disorder, i.e., a reduction of the relative strength of the TO peak (combined with enhanced broadening) upon increasing disorder. This is what we observe in going from Figs. 9(a) to 9(b); here, the TO band is even largely suppressed by the high degree of disorder [Fig. 9(b)]. A dependence upon atomic disorder of the optical phonon peak in the Fe-projected VDOS of crystalline $\mathrm{Pt}_{3} \mathrm{Fe}$ has been also reported by Fultz et al. ${ }^{47}$ With increasing disorder in $\mathrm{Pt}_{3} \mathrm{Fe}$, the optical-phonon VDOS peak at $25 \mathrm{meV}$ underwent a reduction in intensity combined with asymmetric broadening towards higher and lower energies. This observation is qualitatively similar to the changes seen in the TO region of the VDOS in going from Fig. 9(a) to Fig. 9(b), except that the TO peak is nearly entirely suppressed in Fig.

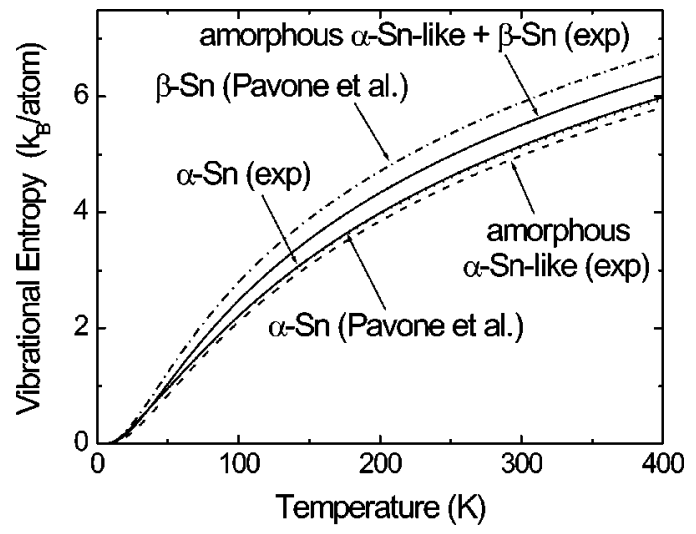

FIG. 11. Temperature dependence of the vibrational entropy of epitaxial $\alpha$-Sn(500 $\AA$ )/InSb(001) (bold solid line), of amorphous$\alpha$-Sn-like $(10 \AA) / a$-Si multilayer (dashed line), and of $\mathrm{Sn}(20 \AA) / a$-Si multilayer (solid line) deduced from the VDOS in Figs. 9(a)-9(c), respectively. Values obtained from the theoretical VDOS of bulk $\alpha$-Sn and $\beta$-Sn are shown for comparison (dotted line and dashed-dotted line, respectively) (Ref. 6).

9(b), presumably by a very high degree of atomic disorder in our $10-\AA ̊$-thick Si-embedded Sn layers.

In this context it is interesting to notice that in the VDOS of $20 \AA$ Sn embedded between $a$-Si [Fig. 9(c)], sharper features than in Fig. 9(b) (for $10 \AA \mathrm{Sn}$ ) appear in the region of the optical phonons of $\alpha$-Sn. For $20 \AA$ Sn [Fig. 9(c)], the cutoff at $25 \mathrm{meV}$ is as sharp as that of $\alpha$-Sn [Fig. 9(a)], and the weaker TO peak near $23 \mathrm{meV}$ and the stronger LO peak near $20 \mathrm{meV}$, both of $\alpha$-Sn, can be clearly detected in Fig. 9(c). This means that according to our interpretation the interfacial amorphous- $\alpha$-Sn-like layers in the $\operatorname{Sn}(20 \AA) / \mathrm{Si}(50$ $\AA)$ multilayer are less disordered than the amorphous Sn layers in the $\operatorname{Sn}(10 \AA) / a$-Si multilayer. Possibly the crystallization process to $\beta$-Sn, which occurs above the critical $\mathrm{Sn}$ thickness of $10 \AA$, lowers the degree of disorder in the residual 10 - $\AA$-thick amorphous- $\alpha$-Sn-like layer at the $a$-Si interface. An alternative explanation for the sharper features in the optical region could be reduced hybridization of $a-\mathrm{Sn} / a-\mathrm{Si}$ phonon modes due to the fact that one of the $a-\mathrm{Sn} / a-\mathrm{Si}$ interfaces is replaced by a $\beta-\mathrm{Sn} / a-\mathrm{Sn}$ interface in the $20 \AA$ Sn sample.

\section{2. $f$ factor and vibrational entropy}

The $f$ factor of the amorphous interfacial $\alpha$-Sn-like layer is found to be enhanced relative to the $f$ factor of crystalline $\alpha$-Sn (Ref. 4 and Table II). The calculation of the $f$ factor strongly weighs the low-energy part of the VDOS, and the observed enhancement originates from the shift towards higher energy of the low-energy TA band of crystalline $\alpha$-Sn [Fig. 9(a)] to the strong $10-\mathrm{meV}$ peak of amorphous $\alpha$-like Sn [Fig. 9(b)].

In Fig. 11 we show the temperature dependence of the vibrational entropy per atom of the crystalline $\alpha$-Sn $(500 \AA) /$ $\mathrm{InSb}(001)$ system and of the amorphous- $\alpha$-like-Sn interface layer, as calculated from the corresponding measured VDOS 
[i.e., $g(E)$ ] and Eq. (8.5) in Ref. 37. Also shown in Fig. 11 is the theoretical vibrational entropy of crystalline (bulk) $\alpha$-Sn and $\beta$-Sn by Pavone et al. ${ }^{6}$ The agreement between the theory and experiment in the case of $\alpha$-Sn is very good suggesting that a 500- $\AA$-thick $\alpha$-Sn film behaves like bulk $\alpha$-Sn with respect to the vibrational dynamics. The vibrational entropy of the $\left[\operatorname{Sn}(20 \AA) / \operatorname{Si}(50 \AA)_{25}\right.$ multilayer, that contains $\beta$-Sn and interfacial amorphous- $\alpha$-like $\mathrm{Sn}$, is also shown in Fig. 11; its entropy is located about midway between the entropy of interfacial amorphous $\alpha$-like $\mathrm{Sn}$ and $\beta$-Sn, as expected from the composition of this sample.

Figure 11 also demonstrates that the vibrational entropy per atom of the 10 - $\AA$-thick amorphous- $\alpha$-Sn-like interface layer is fairly close to that of crystalline $\alpha$-Sn. This gives an answer to the question why a thin metastable amorphous$\alpha$-Sn-like layer is more favorable at the $a$-Si interface at RT than the stable high-temperature $\beta$ phase. The theoretical study by Pavone et al. ${ }^{6}$ for bulk Sn demonstrates that the $\beta$-Sn phase is stabilized at high $T$ (e.g., at $300 \mathrm{~K}$ ) by its larger vibrational entropy, and consequently by its lower free energy ( $F=U-T S$ ), relative to $\alpha$-Sn. Because of the nearly identical vibrational entropies per atom of $\alpha$-Sn and the interfacial amorphous- $\alpha$-like Sn layer (Fig. 11), we might similarly expect the $\beta$ phase to be more stable at high $T$ than the amorphous- $\alpha$-Sn-like structure. However, the observed stability of the interfacial amorphous-Sn layer contradicts this hypothesis. Preliminary high-temperature Mössbauer measurements on our samples demonstrate that this interfacial amorphous-Sn layer is stable at least up to $200^{\circ} \mathrm{C}$. Therefore, it must be the interface free energy between amorphous $\alpha$-Sn and the underlying amorphous-Si film that provides the basis for the observed stabilization of the metastable amorphous- $\alpha$-Sn-like layer, and is not the vibrational entropy. We have to assume that the interfacial free energy of the amorphous- $\alpha$-like $\mathrm{Sn} / a$-Si system is lower than that of the $\beta$-Sn $/ a-\mathrm{Si}$ system in order to understand the metastability of the amorphous-Sn/a-Si system. At $300 \mathrm{~K}$, we have observed a critical film thicknesses of $10 \AA$ below which the amorphous-Sn structure is stable with respect to the crystalline $\beta$ phase (Fig. 4). This phenomenon is reminiscent of the pseudomorphic growth of metastable phases in the form of epitaxial thin films on a single-crystalline substrate. ${ }^{48,49}$ However, the growing amorphous- $\alpha$-Sn-like structure is probably able to accommodate the "lattice" misfit with the $a$-Si film more easily than a crystalline system.

The argument that the $a$-Sn phase is stabilized by surfaceenergy contributions may find additional support from the fact that the a-Sn layer has limited thickness. If entropy should explain the $a$-Sn formation the limited thickness would be difficult to explain, after all vibrational entropy is given per atom. If one would insist that vibrational entropy is responsible, a strong change of the VDOS across the $a$-Sn layer were required. But this leads to a contradiction to our data that show that the average entropy in the $a$-Sn layer is much smaller than that of $\beta$-Sn. It is impossible that regions of high entropy exist in the $a$-Sn layer without also having regions of lower entropy. To continue the line of thought one might suggest that the $a-\mathrm{Si} / a-\mathrm{Sn}$ interface free energy is smaller than the $a-\mathrm{Sn} / \beta$-Sn interface free energy leading to the observed stable $a$-Sn layer.

\section{CONCLUDING REMARKS}

The structure and vibrational dynamics of nanoscale $\mathrm{Sn} / a$-Si multilayers grown at room temperature (RT) in ultrahigh vacuum on $\mathrm{Si}(111)$ wafers have been studied by means of $\mathrm{x}$-ray diffraction (XRD), Raman scattering, ${ }^{119} \mathrm{Sn}$ Mössbauer spectroscopy (CEMS), and ${ }^{119} \mathrm{Sn}$-inelastic nuclear-resonant scattering (NRIXS) of synchrotron radiation. The Raman line shifts observed are typical for amorphous $(a-\mathrm{Si})$. Mössbauer isomer shifts demonstrate that the Sn layers in these multilayers exist in a pure $\alpha$-Sn-like phase up to a Sn thickness $\left(t_{\mathrm{Sn}}\right)$ of $10 \AA$. The formation of crystalline $\beta$-Sn was observed with increasing Sn thickness, with 10 - $\AA$-thick $\alpha$-Sn-like layers remaining stabilized at the $\mathrm{Sn} / \mathrm{Si}$ interfaces at RT. Since no high-angle XRD Bragg peaks typical for crystalline $\alpha$-Sn could be detected, it is likely that the 10 - $\AA \alpha$-Sn-like interfacial layers are amorphous, similar to the underlaying Si layers. By means of NRIXS we have measured the Sn-projected vibrational density of states (VDOS) in $\mathrm{Sn} / a$-Si multilayers with thicknesses $t_{\mathrm{Sn}}=10,20 \AA$ and $t_{\mathrm{Si}}=20,50 \AA$, and in $500-\AA$-thick epitaxial $\alpha$-Sn on $\mathrm{InSb}(001)$ as a reference. The VDOS of the multilayers were found to be distinct from those of the crystalline (bulk) $\alpha$ and $\beta$-Sn phases. Scaling arguments for the VDOS provide further evidence for the amorphous nature of $10-\AA$-thick $\alpha$-Sn-like interfaces.

Further, the Lamb-Mössbauer factor, mean kinetic energy per atom, mean atomic force constant, and the vibrational entropy per atom were obtained. The vibrational entropy deduced from the VDOS of a 500- $\AA$-thick epitaxial $\alpha$-Sn film on $\operatorname{InSb}(001)$ is found to be in good agreement with a recent theory. ${ }^{6}$ At $300 \mathrm{~K}$, the observed small difference in vibrational entropy, $\Delta S / k_{B}$, of $+0.17 \pm 0.05$ per atom between $\alpha$-Sn and interfacial amorphous $\alpha$-like Sn does not account for the stability of the interfacial amorphous-Sn layer. Consequently, it is the amorphous- $\mathrm{Sn} / a$-Si interface free energy that stabilizes the metastable amorphous-Sn phase. In this context it is interesting to mention that a similar behavior in terms of interfacial $\alpha$-Sn layer thickness is observed, if one uses the crystalline $\mathrm{Si}(111)-(7 \times 7)$ surface as template for the growth of epitaxial Sn films. ${ }^{50}$ It appears that the structure and symmetry of the substrate is irrelevant with respect to the thickness of the $\alpha$-Sn layer (amorphous or crystalline) that is stabilized by interface free energy terms.

In addition, our results demonstrate that NRIXS is a unique method for investigating the vibrational dynamics of buried interfaces.

We are grateful to U. von Hörsten for valuable technical assistance and to Dr. M. Grimsditch for cooperation in Raman spectroscopy. This work was supported by the Deutsche Forschungsgemeinschaft (GRK 277 and Ke 273/12-1). Use of the advanced photon source was supported by the U.S. Department of Energy, Basic Energy Science, Office of Science, under Contract No. W-31-109-Eng-38. 
*Email address: roldan@uni-duisburg.de

${ }^{1}$ D. L. Weaire, in Amorphous Solids-Low Temperature Properties, edited by W. A. Phillips, Topics in Current Physics, Vol. 24, (Springer, Berlin, 1981), p. 13, and references cited therein.

${ }^{2}$ M. Cardona, in Phonon Physics, edited by J. Kollár, N. Kroó, N. Menyhárd, and T. Siklós (World Scientific, Singapore, 1985), p. 2 , and references cited therein.

${ }^{3}$ H. Omi, H. Saito, and T. Osaka, Phys. Rev. Lett. 72, 2596 (1994).

${ }^{4}$ L. K. Nanver, G. Weyer, and B. I. Deutch, Z. Phys. B: Condens. Matter 47, 103. (1982).

${ }^{5}$ R. Alben, D. Weaire, J. E. Smith, Jr., and M. H. Brodsky, Phys. Rev. B 11, 2271 (1975); Phys. Rev. Lett. 29, 1505 (1972).

${ }^{6}$ P. Pavone, S. Baroni, and S. Gironcoli, Phys. Rev. B 57, 10421 (1998).

${ }^{7}$ R. F. C. Farrow, D. S. Robertson, G. M. Williams, A. G. Cullis, G. R.Jones, I. M. Young, and P. N. J. Dennis, J. Cryst. Growth 54, 507 (1981).

${ }^{8}$ W. T. Yuen, W. K. Liu, S. N. Holmes, and R. A. Stradling, Semicond. Sci. Technol. 4, 819 (1989).

${ }^{9}$ W. T. Yuen, W. K. Liu, B. A. Joyce, and R. A. Stradling, Semicond. Sci. Technol. 5, 373 (1990).

${ }^{10}$ W. T. Yuen, W. K. Liu, R. A. Stradling, and B. A. Joyce, J. Cryst. Growth 111, 943 (1991).

${ }^{11}$ B. F. Mason and B. R. Williams, Surf. Sci. 262, 169 (1992).

${ }^{12}$ B. Roldan Cuenya, M. Doi, O. Marks, W. Keune, and K. Mibu, in Structure and Dynamics of Heterogeneous Systems, edited by P. Entel and D. E. Wolf (World Scientific, Singapore, 2000), p. 251.

${ }^{13}$ D. T. Wang, N. Esser, M. Cardona, and J. Zegenhagen, Surf. Sci. 343, 31 (1995).

${ }^{14}$ L. Ottaviano, M. Crivellari, L. Lozzi, and S. Santucci, Surf. Sci. Lett. 445, 41 (2000).

${ }^{15}$ C. Törrevik, M. Hammar, N. G. Nilsson, and S. A. Flodström, Phys. Rev. B 44, 13144 (1991).

${ }^{16}$ See, for instance, W. Mönch, Semiconductor Surfaces and Interfaces, Springer Series in Surface Science, Vol. XV (Springer, Berlin, New York 1995).

${ }^{17}$ M. Hansen and K. Anderko, Constitution of Binary Alloys (McGraw-Hill, New York 1958), p. 1193.

${ }^{18}$ M. Seto, Y. Yoda, S. Kikuta, X. W. Zhang, and A. Ando, Phys. Rev. Lett. 74, 3828 (1995).

${ }^{19}$ W. Sturhahn, T. S. Toellner, E. E. Alp, X. W. Zhang, M. Ando, Y. Yoda, S. Kikuta, M. Seto, C. W. Kimball, and B. Dabrowski, Phys. Rev. Lett. 74, 3832 (1995).

${ }^{20}$ W. Keune and W. Sturhahn, Hyperfine Interact. 123/124, 847 (1999).

${ }^{21}$ W. Sturhahn, R. Röhlsberger, E. E. Alp, T. Ruckert, H. Schrör, and W. Keune, J. Magn. Magn. Mater. 198-199, 590 (1999).

${ }^{22}$ R. Röhlsberger, E. E. Alp, E. Gerdau, O. Leupold, K. W. Quast, R. Rüffer, W. Sturhahn, T. S. Toellner, and E. Burkel, Physica B 263-264, 574 (1999).

${ }^{23}$ A. Svane, N. E. Christensen, C. O. Rodriguez, and M. Methfessel, Phys. Rev. B 55, 12572 (1997).
${ }^{24}$ Mössbauer Effect Data Index Covering the 1974 Literature, edited by J. G. Stevens and V. E. Stevens (IFI, New York, 1975), p. 153.

${ }^{25}$ R. A. Brand, Nucl. Instrum. Methods Phys. Res. B 28, 417 (1987).

${ }^{26}$ R. Rüffer and A. I. Chumakov, Hyperfine Interact. 97/98, 589 (1996).

${ }^{27}$ T. S. Toellner (unpublished).

${ }^{28}$ H. Kiessig, Ann. Phys. 10, 769 (1931).

${ }^{29}$ L. G. Parratt, Phys. Rev. B 5, 359 (1954).

${ }^{30}$ D. A. Shirley, M. Kaplan, and P. Axel, Phys. Rev. B 12, 816 (1961).

${ }^{31}$ A. Barla, R. Rüffer, A. I. Chumakov, J. Metge, J. Plessel, and M.M. Abd-Elmeguid, Phys. Rev. B 61, R14 881 (2000).

${ }^{32}$ X. Zhang, J. D. Comins, A. G. Every, P. R. Stoddart, W. Pang, and T. E. Derry, Phys. Rev. B 58, 13677 (1998).

${ }^{33}$ D. Bermejo and M. Cardona, J. Non-Cryst. Solids 32, 405 (1979).

${ }^{34}$ J. Menendez and M. Cardona, Phys. Rev. B 29, 2051 (1984).

${ }^{35} \mathrm{M}$. Hu, Dissertation, Northwestern University, Evanston, Illinois, USA, 1999.

${ }^{36}$ W. Sturhahn, Hyperfine Interact. 125, 149 (2000).

${ }^{37}$ A. I. Chumakov and W. Sturhahn, Hyperfine Interact. 123-124, 781 (1999).

${ }^{38}$ M. Hu, W. Sturhahn, T. S. Toellner, P. M. Hession, J. P. Sutter, and E. E. Alp, Nucl. Instrum. Methods Phys. Res. A 428, 5511 (1999).

${ }^{39}$ W. Sturhahn and A. I. Chumakov, Hyperfine Interact. 123-124, 809 (1999).

${ }^{40}$ M. Fanciulli, H. C. Vestergaard, G. Weyer, M. Fyhn, S. Yu Shiryaev, and A. Nylandsted Larsen, in Proceedings of 23rd International Conference on the Physics of Semiconductors, edited by M. Scheffler and R. Zimmermann (World Scientific, Singapore, 1996), Vol. 2, p. 1059.

${ }^{41}$ U. Gonser, in Mössbauer Spectroscopy, edited by U. Gonser (Springer, Berlin, 1975), p. 1.

${ }^{42}$ W. A. Kamitakahara, H. R. Shanks, J. F. McClelland, U. Buchenau, F. Gompf, and L. Pintschovius, Phys. Rev. Lett. 52, 644 (1984).

${ }^{43}$ R. M. Martin, Phys. Rev. B 1, 4005 (1970).

${ }^{44}$ C. J. Buchenauer, M. Cardona, and F. H. Pollak, Phys. Rev. B 3, 1243 (1971).

${ }^{45}$ R. Tsu, J. González-Hernández, and F. H. Pollak, Solid State Commun. 54, 447 (1985).

${ }^{46}$ J. E. Yehoda and J. S. Lannin, J. Vac. Sci. Technol. A 1, 392 (1983).

${ }^{47}$ B. Fultz, T. A. Stephens, E. E. Alp, M. Y. Hu, J. P. Sutter, T. S. Toellner, and W. Sturhahn, Phys. Rev. B 61, 14517 (2000).

${ }^{48}$ G. Prinz, J. Magn. Magn. Mater. 100, 469 (1991).

${ }^{49}$ E. S. Machlin, An Introduction to Aspects of Thermodynamics and Kinetics Relevant to Materials Science (Giro Press, Crotonon-Hudson, New York, 1991), p. 131.

${ }^{50}$ B. Roldan Cuenya, M. Doi, and W. Keune, Surf. Sci. (to be published). 